\title{
CCD ASTROMETRY OF THE SOLAR SYSTEM
}

\author{
J.-E. ARLOT AND F. COLAS \\ Bureau des Longitudes \\ Paris, France
}

\section{Introduction}

Astrometry in the solar system made more progress during the past ten years than it did during the seven or eight decades before. In fact, the development of large refractors with micrometers, and after the improvement of the photographic technique, allowed one to get very accurate positions at the end of the $19^{\text {th }}$ century. After that, relatively little progress was made. Astronomers faced several difficulties: first, was the poor sensitivity of the photographic plates leading to long exposures and therefore to low accuracy for faint objects; second, was the poor quality of the catalogues of stars which depended upon a very small number of available astrometric reference stars.

New receptors appeared around the 1950 , such as electronic cameras or T.V. tubes. But, most of these new receptors were not interesting for astrometry, mainly because of the field of view - which was too small and of the difficulty of using such receptors. However, the development of a new technique based upon the CCD targets changed most of the astrometric observational programs. In fact, $C C D$ receptors were made for small fields, but the CCDs were easy to use; the images were numerical, allowing processing by computers, and the sensitivity was much more important. Because of that, the accuracy increased and relative astrometry was developed. The recent development of new catalogues, such as the Guide Star Catalogue and Tycho, permits astronomers to reduce small fields, which was not possible earlier.

\section{Long Focus CCD Astrometry}

In this section, we summarize the results obtained with small field astrometry due to the association of long focus telescopes with CCD receptors. The 
first applications of CCD to astrometric observations of the solar system, concerned the planetary satellites. Those objects need only a small field to be observed since they are close together.

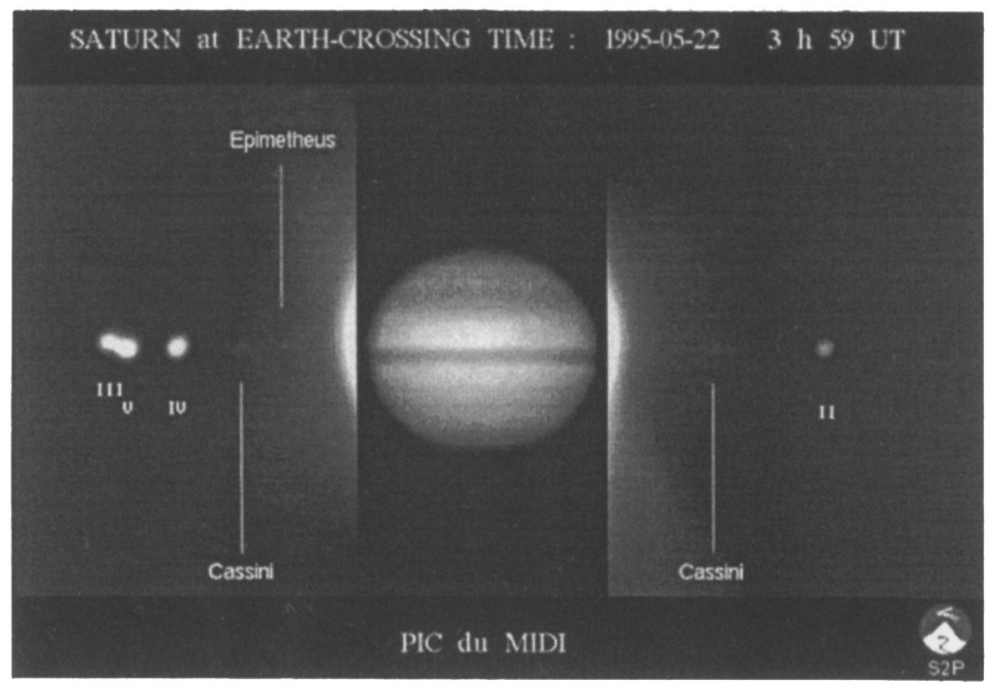

Figure 1. This CCD image of Saturn and its satellites has been made at Pic du Midi during the ring plane crossing of 1995.

In fact, photographic observations of planetary satellites provided rather accurate relative positions since long focus refractors or reflectors were used. However, the main advantage of CCDs is their ability to observe faint satellites thanks to their sensitivity. Unfortunately, most of the faint planetary satellites are very close to their planet which is often very bright. This difficulty was solved by using several methods in order to decrease the brightness of the primary.

Three methods are commonly used: first, the re-imaging coronograph as used at Flagstaff by the USNO; second, a neutral density filter as used for example at La Palma on the Jacobus Kapteyn telescope; and third, an antiblooming CCD as used by Bureau des longitudes at Pic du Midi Observatory and at ESO in Chile. These techniques allowed astronomers to observe faint satellites which were unable to be observed easily before: the satellites of Mars, the faint satellites of Jupiter close to the planet and the faint satellites of Neptune, such as Proteus, which were observed first in La Silla, Chile. During the Earth-crossing of the Saturnian ring plane last year, it was also possible to observe faint satellites of Saturn such as Epimetheus or Helene.

However these types of long-focus observation were improved. First, comes the use of infrared arrays, as done by Phil Nicholson and colleagues 


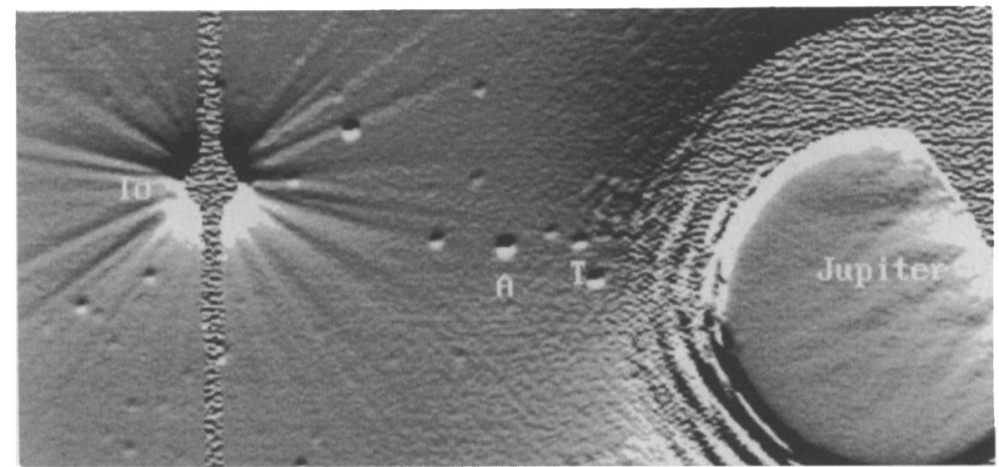

Figure 2. This image from LNA in Brazil shows the satellite Thebe of Jupiter. The use of antiblooming CCD is necessary because of the proximity of the bright planet.

at Palomar Observatory at 2.2 microns, wherein the brightness of the planet is decreased by the methane absortion of the giant planet's atmosphere: since the satellite light is not absorbed they are easier to observe. Second comes the adaptive optics used, for example, by André Brahic at the CFHT in Hawaii and by Bruno Sicardy at ESO, Chile. However, the best results for faint satellites are obtained by the HST and the satellites discovered by Voyager, which were observed and analyzed by Pascu and colleagues.

Concerning the reduction techniques, it is important to bear in mind that it is only possible to make an astrometrical reduction if two objects are present in the field of view.

First, as it was done by Pascu for the Uranian moons and by Colas for the faint satellite Proteus of Neptune, it is possible to use the positions of well-known planetary satellites in order to reduce the observations of the fainter satellites.

Second, the use of external calibration fields such as double or multiple stars or globular clusters gave good results for the Martian moons leading to an accuracy of 0.1 arcsecond in relative positions with a calibration field accurate to 0.04 arcsecond. This method was used by Jones and colleagues at La Palma and by Colas and Arlot at Pic du Midi Observatory.

The third method to be mentioned is the one used by Veiga and Vieira Martins: the well-known slow motion of the planet observed night after night allows one to calibrate the field by building of a local reference catalogue.

However, even with a small field 1 to 10 arc-minutes in size, absolute astrometry is possible. Let us consider the observation of asteroids. The 
coming of CCDs with small fields permits a new type of observation: the observation of close approaches on the sky of asteroids and stars. These observations allow one to get very accurate positions of the asteroids depending on the accuracy of the position of the star. In that case, the motion of the asteroid will help to calibrate the field. Moreover, when an occultation of the star is observed, the accuracy of the determination of the position of the asteroid is of the order of a few milliarseconds. CCDs allow one to get a large number of frames during these close approaches, which increases the accuracy. These can also be applied to calibrate a field as was done for Charon observation by Young et al. (1994).

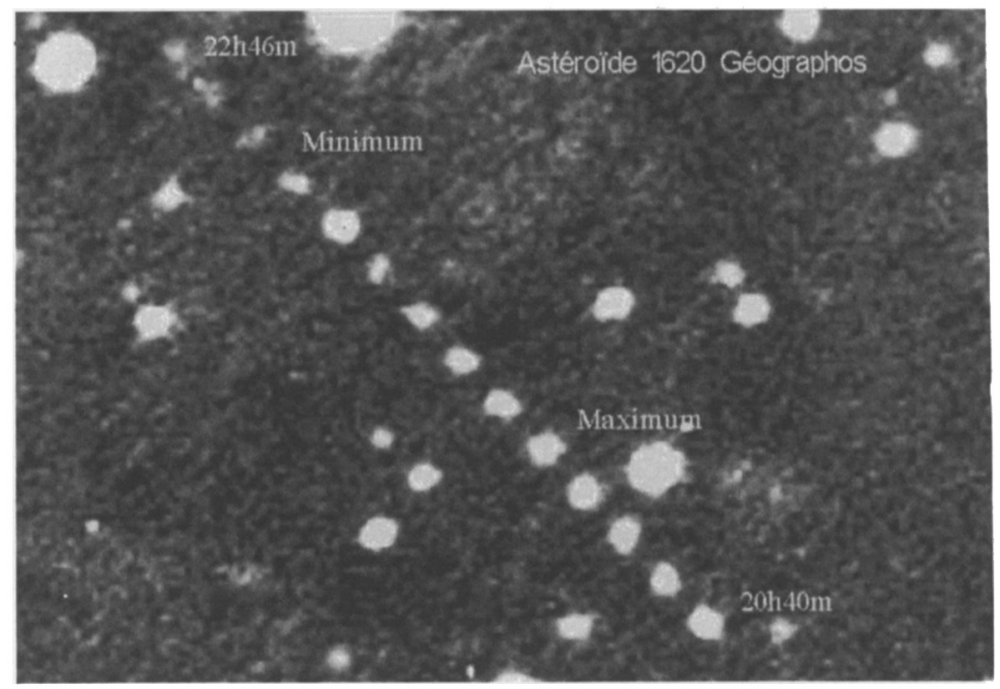

Figure 3. On this composite CCD image from Observatoire de Haute Provence, we see the path of Geographos in front of the field of stars. The motion of Geographos will help to make the astrometric reduction of this small field.

Observations of comets benefit also from the use of CCDs. In that case, the main problem comes frome the fact that comets may not be considered as a point source. In order to determine how their center of mass differs from the center of light, it is necessary to study their figure, which is possible with CCDs associated with a long focus telescope. In fact, the same problem occurs with the large objects of the solar system. The problems related to the surface of the planets is well known, even if not yet well-solved, and the same problem exists for planetary satellites. CCDs associated with long focus telescopes allow one to get surface features of some objects such as Ganymede. Adaptive optics will increase these possibilities. 
What is the contribution of CCDs in planet observations? We will see further that meridian transit circles get a large benefit from using CCDs as a new receptor. However, is the direct observation of planets possible with CCDs? We know that their field will never reach the size of photographic plates. In that case, it is interesting to use the new star catalogues, such as the Tycho catalogue, which contain a large number of stars and to select the period of observation when stars are present in the field near the planet. In order to increase the accuracy of the observed positions, it is especially interesting to measure the positions of the satellites around the planet and to deduce from their positions, the location of the planet. We do not need a lot of stars since the scale and the orientation of the field may be determined as previously. In conclusion, it is the conjunction of the development of CCDs and of the coming of new accurate astrometric catalogues of stars which will be a benefit for the astrometry of the solar system.

\section{Two Dimensional Photometry}

We would like now to come to the indirect astrometry using photometric data. The goal is to measure events such as the eclipses of the Galilean satellites and to deduce astrometric data as the position of the objects in their orbits. Photographic plates were difficult to use for that purpose because of the non-linear response of the receptor and because of the delay between two exposures. Photoelectric photometers were not easy to use because of the need of several photometric references, leading to multichannel photometers which are difficult to build and to manage. CCDs allow a two dimensional photometry so that the reduction is easy, and events not obtainable before are now observed - such as the phenomena of the satellites of Saturn: eclipses by Saturn, mutual events in spite of the brightness of the ring. Moreover, during such events, the nature of the features on the surfaces of the satellites are detectable, and then the centers of mass are determined with a higher accuracy. When observing simultaneously in several spectral bands, the astrometric reduction of the light curve will allow the determination of the position on the surface of a satellite. In order to avoid biases, simultaneous observations are necessary in several, spectral bands, at least IR and V.

\section{Large Fields}

Now, let us come to the possibility of observing large fields using CCDs. However, it is important to explain the meaning of 'large field' here. We don't think of absolute and global astrometry such as transit circles or Hipparcos satellite observations. We just wish to talk about fields larger than a few minutes of arc. Nowadays, the $1 \mathrm{k} \times 1 \mathrm{k}$ pixels CCDs and the $2 \mathrm{k} \times 2 \mathrm{k}$ 
pixels CCDs become very common, so a CCD field of ten minutes of arc can now be considered as a standard for modern telescopes. Moreover, mosaics of several CCDs targets are now available such as the $8 \mathrm{k} \times 8 \mathrm{k}$ camera of the University of Hawaii made of a grid of sixteen $2 \mathrm{k} \times 2 \mathrm{k}$ CCDs. The field of this camera reaches thirty minutes of arc at the CFHT prime focus. With this kind of mosaic, most of a telescope's prime focus can be refurbished in order to reach $26^{\text {th }}$ magnitude stars in a one-degree field containing a few Hipparcos stars and a lot of GSC and Tycho stars. This kind of instruments opens new applications for solar system astrometry: very faint objects such as comets and Kuiper's belt bodies are observable. Moreover, catalogues such as Tycho may be helpful to increase the accuracy of the observations from a few tenths of a second of arc to a few hundredths. Great progress will alsa be possible for comets, for which the position residuals are, at the present time, near one arcsecond.
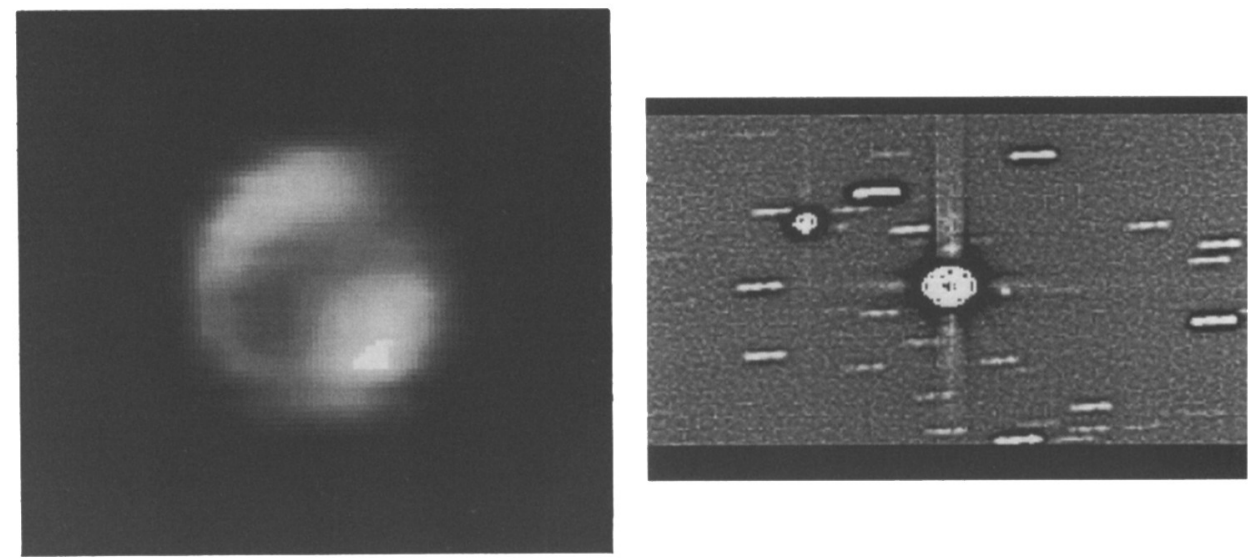

Figure 4. Left: This view of Ganymede from Pic du Midi shows features on the surface of the satellites. This has to be modelized in order to know the center of mass with a higher accuracy.

Right: This image from ESO $2.2 \mathrm{~m}$ telescope shows the first Earth based detection of the satellite Proteus of Neptune.

Let us come now to the new scanning targets. The idea is to scan the sky with a telescope - instead of scanning an image - that will allow an effective increase of the size of the field.

The first idea is to use the good old transit circles, which are in fact the first scanning receptors working with only one pixel! They were first upgraded with photoelectrical photometers in order to remove the bias due to the observer. Then came the idea of using a two-dimensional receptor like CCDs, not only because of its high dynamic and its good quantum efficiency, but also because of its ability to read the chip at the same rate as the motion of the sky. The limit of this technique comes from the fact 
that the motion of the sky does not have a constant rate, but it depends upon the declination of the field observed. It is necessary to use fields smaller than 30 arc-minutes at a declination not higher than sixty degrees. It is easy to see the high interest of this technique: first, a meridian circle able to reach magnitude thirteen with the photoelectric photometer, such as for example the meridian circle of Bordeaux Observatory, will then reach magnitude seventeen; second, a one-hour observation will provide a strip of half a degree by fifteen degrees, containing between fifty and one hundred Tycho stars. At the present time three meridian circles are equipped with CCDs in Bordeaux, France, Valinhos, Brasil and in Flagstaff at the USNO station, and others, such as in Pulkovo, are nearly equipped. They are now able to link secondary star catalogues to the Tycho and Hipparcos reference frames. You may see the interest of these secondary catalogues for the users of standard small-field CCDs and for the reduction of the observations of comets and asteroids. The scanning machine may also be a standard telescope stopped in a given position. With this technique, problems of guiding and pointing are solved. This technique, as employed by Spacewatch at Kitt Peak, is used to find fast-moving objects by scanning a strip of sky two times in one night. Spacewatch used a one meter and a half telescope and discovers objects of magnitude 20. At Pic du Midi Observatory, we are refurbishing a $55 \mathrm{~cm}$ telescope with a CCD covering a field of a half-degree at the prime focus, and we use three correcting lenses. The pixel is about one arcsecond. The telescope will be operating either in standard mode with a guiding star, or in the scanning mode. The goal is to connect large fields, containing many astrometric stars obtained in the scanning mode, with deep fields until magnitude 23 obtained in the tracking mode.

Other techniques are also used in order to get large fields with CCD: first is the scanning of the sky along great circles which permits the scanning of the sky at high declinations. Second is the moving of a CCD target in the focal plane of the telescope, as proposed by Yershov and Lopez Garcia in the poster presented at the present colloquium.

Finally, speaking of larger fields leads us to talk about Schmidt telescopes. After the photographic-plate era, these telescopes will have a new life, even if thirty centimeters large CCDs are not yet available. They will benefit from mosaics of CCDs covering one third of the focal surface. So, three exposures will cover the whole field, and nine exposures will cover the field for a three-color survey. New problems will occur, but these telescopes, together with other techniques, should make possible an extensive search for small solar system objects, with an accuracy sufficient to calculate accurate orbits allowing the study of their evolution. The OCA Schmidt telescope will be refurbished for example with sixteen $2 \mathrm{k} \times 2 \mathrm{k}$ CCDs. 


\section{Conclusion}

It is clear that we are entering into a new astrometrical era, mainly due to progress in hardware, such as receptors or computers. Moreover, the availability of high quality astrometrical catalogues such as Tycho will solve the main drawback of small field CCD observations. New research domains are now open with the possibility to have high astrometrical accuracy for faint objects like comets or Kuiper Belt objects. But, we have to face up to the fact that there is a giant amount of data that we have to archive; we learned from the past that observations made even decades earlier are sometimes very useful.

\section{References}

Arlot, J.-E. and Thuillot, W.: 1996, Proceedings of the workshop "PHESAT95" held in Bucharest, Romania, Sept. 1994, Supplément aux Annales de Physique 21.

Colas, F. and Arlot, J.-E.: 1991, "Comparisons of observations of the Martian satellites made in 1988 with ephemerides", Astron. Astrophys. 252, 402.

Colas, J.F. and Buil, C.: 1992, "First Earth-based observations of Neptune's satellite Proteus", Astron. Astrophys. 262, L13.

Jones, D.H.P.: 1996, "Limitations on the accuracy possible in astrometric observations of satellites of the major planets" in: Dynamics, Ephemerides and Astrometry of the Solar System, IAU Symposium 172 (S. Ferraz-Mello, B. Morando, J.-E. Arlot, eds), Kluwer, Dordrecht.

Nicholson, P.D. and Matthews, K.: 1991, "Near-infrared observations of the Jovian ring and small satellites", Icarus $93,331$.

Nicholson, P.D., Hamilton, D.P., Matthews, K., and Yoder, C.F.: 1992, "New observations of Saturn's coorbital satellites", Icarus 100, 464.

Pascu, D., Seidelmann, P.K., Baum, W.A., and Schmidt, R.E.: 1983, "Observations of faint planetary satellites with a charge-coupled device", in: The Motion of Planets and Natural and Artificial Satellites (S. Ferraz-Mello, P.E. Nacozy, eds).

Pascu, D., Rohde, J.R., Seidelmann, P.K., Currie, D.G., Dowling, D.M. Wells, E., Kowal, C., Zellner, B., and Storrs, A.: 1995, "HST astrometry of the Uranian inner satellite system", Bull. Am. Astron. Soc. 27, 829.

Veiga, C.H. and Vieira Martins, R.: 1996, "A method to define a reference system for the reduction of astrometric positions of natural satellites", Astron. Astrophys., Suppl. Ser. 107, 551.

Veiga, C.H. and Vieira Martins, R.: 1996, "CCD observations of Triton", Astron. Astrophys., in press.

Young E.F. and Binzel, R.P.: 1993, "Comparative mapping of Pluto's sub-Charon hemisphere: Three least squares models based on mutual event light curves", Icarus 102, 134.

Young L.A., Olkin, C.B., and Elliot, J.L.: 1994, "The Charon-Pluto mass ratio from MKO astrometry", Icarus 108, 186. 